\title{
HODGE CYCLES ON KUGA FIBER VARIETIES
}

\author{
MIN HO LEE
}

(Received 22 April 1993)

Communicated by H. R. Rubinstein

\begin{abstract}
We determine the dimension of the space of Hodge cycles for the generic fibers of the Kuga fiber varieties associated to certain quaternion algebras.
\end{abstract}

1991 Mathematics subject classification (Amer. Math. Soc.): 14C30, 14K10.

\section{Introduction}

One of the well-known conjectures in algebraic geometry is the Hodge conjecture which states that every Hodge cycle on a complex projective variety is an algebraic cycle. In this paper, we consider Hodge cycles on generic fibers of certain Kuga fiber varieties.

Let $V$ be a vector space of dimension $2 n$ over $\mathbb{Q}$, and let $L$ be a lattice in $V$. Let $\beta$ be a nondegenerate alternating bilinear form on $V$ such that $\beta(L, L) \subset \mathbb{Z}$. Let $S p(V, \beta)$ be the symplectic group of the pair $(V, \beta)$, and let $\mathscr{H}$ denote the Siegel half space determined by $\beta$ (see Section 4$)$. Then each element $J \in \mathscr{H}$ defines a complex structure on $V(\mathbb{R})$ and there is a unique complex analytic structure on $\mathscr{H} \times V(\mathbb{R})$ such that the natural projection $\mathscr{H} \times V(\mathbb{R}) \rightarrow \mathscr{H}$ is a complex vector bundle over $\mathscr{H}$. For each $J$, if we denote the complex vector space $(V(\mathbb{R}), J)$ by $V_{J}$, then the complex torus $V_{J} / L$ is an abelian variety with polarization $\beta$. Let $A_{\mathscr{H}}$ denote the quotient space $L \backslash \mathscr{H} \times V(\mathbb{R})$, where $L$ acts on $\mathscr{H} \times V(\mathbb{R})$ by $l \cdot(J, v)=(J, v+l)$ for $J \in \mathscr{H}, v \in V(\mathbb{R})$ and $l \in L$. The projection map $\mathscr{H} \times V(\mathbb{R}) \rightarrow \mathscr{H}$ induces the fiber bundle $\pi_{\mathscr{H}}: A_{\mathscr{H}} \rightarrow \mathscr{H}$ whose fibers are abelian varieties isomorphic to $V_{J} / L$ polarized by $\beta$. Let $S p(L, \beta)$ be the subgroup of $S p(V, \beta)$ of elements $g$ with $g L=L$, and take a subgroup $\Gamma_{0}$ of $\operatorname{Sp}(L, \beta)$ of finite index that contains no

(C) 1996 Australian Mathematical Society $0263-6115 / 96 \$ A 2.00+0.00$ 
elements of finite order. Then the quotient $X_{0}=\Gamma_{0} \backslash \mathscr{H}$ is an arithmetic variety that can be considered as a Zariski open subset of a complex projective variety. Now the fiber bundle $\pi_{\mathscr{H}}: A_{\mathscr{H}} \rightarrow \mathscr{H}$ induces the standard family of abelian varieties $\pi_{0}: Y_{0} \rightarrow X_{0}$ over $X_{0}$ (see for example [4, 8], [9, Chapter 4]).

Let $\tilde{G}$ be a semisimple algebraic group defined over $\mathbb{Q}$, and let $\tilde{K}$ be a maximal compact subgroup of the semisimple Lie group $\tilde{G}(\mathbb{R})$. We assume that the symmetric space $\tilde{D}=\tilde{G}(\mathbb{R}) / \tilde{K}$ has a $\tilde{G}(\mathbb{R})$-invariant complex structure. Let $\tilde{\Gamma} \subset \tilde{G}(\mathbb{Q})$ be a torsion-free cocompact arithmetic subgroup of $\tilde{G}$, and let $\tilde{X}=\tilde{\Gamma} \backslash \tilde{D}$ be th: corresponding arithmetic variety. Let $\tilde{\rho}: \tilde{G} \rightarrow S p(V, \beta)$ be a homomorphism, and let $\tilde{\tau}: \tilde{D} \rightarrow \mathscr{H}$ be a holomorphic map such that $\tilde{\rho}(\tilde{\Gamma}) \subset \Gamma_{0}$ and $\tilde{\tau}(\tilde{g} \tilde{y})=\tilde{\rho}(\tilde{g}) \tilde{\tau}(\tilde{y})$ for all $\tilde{g} \in \tilde{G}(\mathbb{R})$ and $\tilde{y} \in \tilde{D}$. Then the pair $(\tilde{\rho}, \tilde{\tau})$ determines a fiber variety $\tilde{\pi}: \tilde{Y} \rightarrow \tilde{X}$ called a Kuga fiber variety over the arithmetic variety $\tilde{X}$ whose fibers are abelian varieties. Such a Kuga fiber variety can be constructed as follows. The semidirect product $\tilde{\Gamma} \ltimes_{\tilde{\rho}} L$ with respect to the representation $\tilde{\rho}: \tilde{\Gamma} \rightarrow \operatorname{Aut}(L)$ operates on the product manifold $\tilde{D} \times V(\mathbb{R})$ properly discontinuously by $(\gamma, l) \cdot(y, v)=(\gamma y, \gamma v+l)$ for $(\gamma, l) \in \tilde{\Gamma} \ltimes_{\rho} L$ and $(y, v) \in \tilde{D} \times V(\mathbb{R})$. We set $\tilde{Y}=\tilde{\Gamma} \ltimes_{\rho} L \backslash \tilde{D} \times V(\mathbb{R})$, and denote by $\tilde{\pi}$ the natural projection of $\tilde{Y}$ onto $\tilde{X}=\tilde{\Gamma} \backslash \tilde{D}$. Then $\tilde{\pi}: \tilde{Y} \rightarrow \tilde{X}$ is a fiber bundle over $\tilde{X}$, which is in fact the pullback of the standard fiber bundle $\pi_{0}: Y_{0} \rightarrow X_{0}$ over $X_{0}=\Gamma_{0} \backslash \mathscr{H}$ via the map $\tilde{X} \rightarrow X_{0}$ induced by $\tilde{\tau}: \tilde{D} \rightarrow \mathscr{H}$.

Let $K$ be a totally real number field with $[K: \mathbb{Q}]=m$, and let $S=\left\{\varphi_{1}, \ldots, \varphi_{m}\right\}$ be the set of embeddings of $K$ into $\mathbb{R}$. Let $B$ be a quaternion algebra over $K$, and let $G$ be the algebraic group $\operatorname{Res}_{K / \mathbb{Q}}\left(S L_{1}(B)\right)$ over $\mathbb{Q}$, where Res is the Weil restriction map. Then $G(\mathbb{C})$ can be identified with $S L_{2}(\mathbb{C})^{m}$. We denote by $\rho_{j}$ be the projection of $G(\mathbb{C})$ onto the $j$ th factor of $S L_{2}(\mathbb{C})^{m}$. We fix a subset $R=\left\{\varphi_{a}, \varphi_{b}, \varphi_{c}, \varphi_{d}\right\}$ of $S$, and define the representation $\rho: G(\mathbb{C}) \rightarrow S L_{16}(\mathbb{C}) \subset S p(8, \mathbb{C})$ to be the tensor product $\rho_{a} \otimes \rho_{b} \otimes \rho_{c} \otimes \rho_{d}$. Let $\Gamma$ be a torsion-free arithmetic subgroup of $G$, and let $\tau: D \rightarrow \mathscr{H}$ a holomorphic map such that $\tau(g y)=\rho(g) \tau(y)$ for $y \in D$ and $g \in G(\mathbb{R})$, where $D$ is the quotient of $G(\mathbb{R})$ by a maximal compact subgroup. Let $\pi: Y \rightarrow X$ be the Kuga fiber variety over $X$ determined by the pair $(\rho, \tau)$. Given a point $x \in X$, we denote by

$$
H H^{2 k}\left(Y_{x}, \mathbb{Q}\right)=H^{(k, k)}\left(Y_{x}\right) \cap H^{2 k}\left(Y_{x}, \mathbb{Q}\right)
$$

the space of Hodge cycles in the fiber $Y_{x}$ over $x \in X$. Such Hodge cycles have been studied in a number of papers (see for example $[1,3,5,6,7,10]$ ). The purpose of this paper is to determine the dimension of the space $H H^{2 k}\left(Y_{x}, \mathbb{Q}\right)$ for $0 \leq k \leq 8$ for a generic point $x$ in $X$.

\section{Representations determined by quaternion algebras}

In this section we state a theorem which determines exterior powers of the representation of a complex Lie group associated to a quaternion algebra. Let $K$ be a 
totally real number field with $[K: \mathbb{Q}]=m$, and let $B$ be a quaternion algebra over $K$. Let $S=\left\{\varphi_{1}, \ldots, \varphi_{m}\right\}$ be the set of all embeddings of $K$ into $\mathbb{R}$, and let $K_{j}$ be the completion of $K$ by the embedding $\varphi_{j}: K \hookrightarrow \mathbb{R}$ for each $j \in\{1, \ldots, m\}$. Then the algebra $B \otimes_{K} K_{j}$ is isomorphic to either the algebra $M_{2}$ ( $\mathbb{R}$ ) of $2 \times 2$ real matrices or the Hamiltonian quaternion $\mathbb{H}$. We denote by $S_{0}$ the set of mappings $\varphi_{j}$ with $B \otimes_{K} K_{j} \cong M_{2}(\mathbb{R})$ and for later purposes assume that $S_{0}=\{1, \ldots, n\}$ with $1 \leq n \leq m-3$.

Let $\mathbb{B}=\operatorname{Res}_{K / \mathbb{Q}}(B)$, where Res is Weil's restriction operator. Then we have

$$
\mathbb{B} \otimes_{\mathbb{Q}} \mathbb{R} \cong \prod_{j=1}^{m}\left(B \otimes_{K} K_{j}\right) \cong M_{2}(\mathbb{R})^{n} \times \mathbb{H}^{m-n}
$$

As a ring, $\mathbb{B}$ is isomorphic to $B$. We fix a ring isomorphism $\iota: B \rightarrow \mathbb{B}$. We identify $\mathbb{B} \otimes_{\mathbb{Q}} \mathbb{R}$ with $M_{2}(\mathbb{R})^{n} \times \mathbb{H}^{m-n}$ and denote by $\operatorname{Pr}_{j}$ its projection map onto the $j$ th factor for $1 \leq j \leq m$. Then $\operatorname{Pr}_{j} \circ \iota$ is an isomorphism of $B$ onto $M_{2}(\mathbb{R})$ for $1 \leq j \leq n$ and

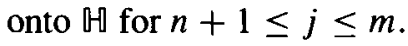

Let $G$ be the algebraic group $\operatorname{Res}_{K / \mathbb{Q}}\left(S L_{1}(B)\right)$ over $\mathbb{Q}$. Then we have

$$
G(\mathbb{Q}) \cong B_{1}^{\times}=\left\{x \in B^{\times} \mid \nu(x)=1\right\}
$$

where $\nu$ is the reduced norm of the quaternion algebra $B$. We identify $G(\mathbb{Q})$ with the subgroup $\iota\left(B_{1}^{\times}\right)$of $\mathbb{B}^{\times}$. Then the Lie group $G(\mathbb{R})$ can be identified with the subgroup $S L_{2}(\mathbb{R})^{n} \times\left(\mathbb{H}_{1}^{\times}\right)^{m-n}$ of $(\mathbb{B} \otimes \mathbb{B})^{\times}=\left(M_{2}(\mathbb{R})^{n} \times \mathbb{H}^{m-n}\right)^{\times}$and $G(\mathbb{C})$ can be identified with $S L_{2}(\mathbb{C})^{m}$. If we also identify $\mathbb{H}_{1}^{\times}$with $S U_{2}$, we have

$$
G(\mathbb{Q}) \subset G(\mathbb{R})=S L_{2}(\mathbb{R})^{n} \times S U_{2}^{m-n} \subset G(\mathbb{C})=S L_{2}(\mathbb{C})^{m}
$$

Let $R=\left\{\varphi_{a}, \varphi_{b}, \varphi_{c}, \varphi_{d}\right\}$ be a subset of $S$ with $|R|=4$. We associate to $R$ a representation $\rho_{R}$ of $G(\mathbb{C})=S L_{2}(\mathbb{C})^{m}$ to $S L_{16}(\mathbb{C})$ by $\rho_{R}=\rho_{a} \otimes \rho_{b} \otimes \rho_{c} \otimes \rho_{d}$, where $\rho_{j}$ is the projection onto the $j$ th factor of $S L_{2}(\mathbb{C})^{m}$ for $1 \leq j \leq m$. We shall denote the representation $\rho_{R}$ with $R=\left\{\varphi_{a}, \varphi_{b}, \varphi_{c}, \varphi_{d}\right\}$ simply by abcd. We shall also denote by $a_{k}$, for example, the $k$ th symmetric power $S^{k}\left(\rho_{a}\right)$ of $\rho_{a}$, and denote the tensor product operation $\otimes$ by $\cdot$ and the direct sum operation $\oplus$ by + respectively.

THEOREM 1. Given the set of embeddings $S=\left\{\varphi_{1}, \ldots, \varphi_{m}\right\}$ of $K$ into $\mathbb{R}$ and $a$ subset $R=\left\{\varphi_{a}, \varphi_{b}, \varphi_{c}, \varphi_{d}\right\}$ of $S$, let $\rho_{R}$ be the representation $\rho_{R}=$ abcd $=\rho_{a} \otimes \rho_{b} \otimes$ $\rho_{c} \otimes \rho_{d}$ of $G(\mathbb{C})=S L_{2}(\mathbb{C})^{m}$ in the complex vector space $\mathbb{C}^{16}$ for $1 \leq a, b, c, d \leq m$ as described above. If $\rho_{U}$ denotes the representation of the compact real form $\left(S U_{2}\right)^{m}$ of $G(\mathbb{C})=S L_{2}(\mathbb{C})^{m}$ induced by $\rho_{R}$, then up to equivalence of representations the exterior powers $\wedge^{k}\left(\rho_{U}\right)$ for $0 \leq k \leq 16$ are as follows: 


$$
\begin{aligned}
& \wedge^{0}\left(\rho_{U}\right)=\wedge^{16}\left(\rho_{U}\right)=1 \text {, } \\
& \wedge^{1}\left(\rho_{U}\right)=\wedge^{15}\left(\rho_{U}\right)=a b c d \text {, } \\
& \wedge^{2}\left(\rho_{U}\right)=\wedge^{14}\left(\rho_{U}\right)=a_{2} b_{2} c_{2}+a_{2} b_{2} d_{2}+a_{2} c_{2} d_{2}+b_{2} c_{2} d_{2}+a_{2}+b_{2}+c_{2}+d_{2} \text {, } \\
& \wedge^{3}\left(\rho_{U}\right)=\wedge^{13}\left(\rho_{U}\right)=a_{3} b_{3} c d+a_{3} b c_{3} d+a_{3} b c d_{3}+a_{3} b c d+a b_{3} c_{3} d+a b_{3} c d_{3} \\
& +a b_{3} c d+a b c_{3} d_{3}+a b c_{3} d+a b c d_{3}+3 a b c d \text {, } \\
& \wedge^{4}\left(\rho_{U}\right)=\wedge^{12}\left(\rho_{U}\right)=3 a_{2} b_{2} c_{2} d_{2}+a_{2} b_{2} c_{2} d_{4}+2 a_{2} b_{2} c_{2}+a_{2} b_{2} c_{4} d_{2}+a_{2} b_{2} c_{4} \\
& +2 a_{2} b_{2} d_{2}+a_{2} b_{2} d_{4}+2 a_{2} b_{2}+a_{2} b_{4} c_{2} d_{2}+a_{2} b_{4} c_{2}+a_{2} b_{4} d_{2} \\
& +2 a_{2} c_{2} d_{2}+a_{2} c_{2} d_{4}+2 a_{2} c_{2}+a_{2} c_{4} d_{2}+2 a_{2} d_{2}+a_{4} b_{2} c_{2} d_{2} \\
& +a_{4} b_{2} c_{2}+a_{4} b_{2} d_{2}+a_{4} b_{4}+a_{4} c_{2} d_{2}+a_{4} c_{4}+a_{4} d_{4}+a_{4} \\
& +2 b_{2} c_{2} d_{2}+b_{2} c_{2} d_{4}+2 b_{2} c_{2}+b_{2} c_{4} d_{2}+2 b_{2} d_{2}+b_{4} c_{2} d_{2}+b_{4} c_{4} \\
& +b_{4} d_{4}+b_{4}+2 c_{2} d_{2}+c_{4} d_{4}+c_{4}+d_{4}+3 \text {, } \\
& \wedge^{5}\left(\rho_{U}\right)=\wedge^{11}\left(\rho_{U}\right)=5 a b c d+4 a b c d_{3}+a b c d_{5}+4 a b c_{3} d+3 a b c_{3} d_{3}+a b c_{3} d_{5} \\
& +a b c_{5} d+a b c_{5} d_{3}+4 a b_{3} c d+3 a b_{3} c d_{3}+a b_{3} c d_{5}+3 a b_{3} c_{3} d \\
& +2 a b_{3} c_{3} d_{3}+a b_{3} c_{5} d+a b_{5} c d+a b_{5} c d_{3}+a b_{5} c_{3} d+4 a_{3} b c d \\
& +3 a_{3} b c d_{3}+a_{3} b c d_{5}+3 a_{3} b c_{3} d+2 a_{3} b c_{3} d_{3}+a_{3} b c_{5} d \\
& +3 a_{3} b_{3} c d+2 a_{3} b_{3} c d_{3}+2 a_{3} b_{3} c_{3} d+a_{3} b_{3} c_{3} d_{3}+a_{3} b_{5} c d \\
& +a_{5} b c d+a_{5} b c d_{3}+a_{5} b c_{3} d+a_{5} b_{3} c d \text {, } \\
& \wedge^{6}\left(\rho_{U}\right)=\wedge^{10}\left(\rho_{U}\right)=6 a_{2} b_{2} c_{2} d_{2}+4 a_{2} b_{2} c_{2} d_{4}+6 a_{2} b_{2} c_{2}+4 a_{2} b_{2} c_{4} d_{2}+a_{2} b_{2} c_{4} d_{4} \\
& +2 a_{2} b_{2} c_{4}+a_{2} b_{2} c_{6}+6 a_{2} b_{2} d_{2}+2 a_{2} b_{2} d_{4}+a_{2} b_{2} d_{6}+a_{2} b_{2} \\
& +4 a_{2} b_{4} c_{2} d_{2}+a_{2} b_{4} c_{2} d_{4}+2 a_{2} b_{4} c_{2}+a_{2} b_{4} c_{4} d_{2}+a_{2} b_{4} c_{4} \\
& +2 a_{2} b_{4} d_{2}+a_{2} b_{4} d_{4}+2 a_{2} b_{4}+a_{2} b_{6} c_{2}+a_{2} b_{6} d_{2}+6 a_{2} c_{2} d_{2} \\
& +2 a_{2} c_{2} d_{4}+a_{2} c_{2} d_{6}+a_{2} c_{2}+2 a_{2} c_{4} d_{2}+a_{2} c_{4} d_{4}+2 a_{2} c_{4} \\
& +a_{2} c_{6} d_{2}+a_{2} d_{2}+2 a_{2} d_{4}+3 a_{2}+4 a_{4} b_{2} c_{2} d_{2}+a_{4} b_{2} c_{2} d_{4} \\
& +2 a_{4} b_{2} c_{2}+a_{4} b_{2} c_{4} d_{2}+a_{4} b_{2} c_{4}+2 a_{4} b_{2} d_{2}+a_{4} b_{2} d_{4}+2 a_{4} b_{2} \\
& +a_{4} b_{4} c_{2} d_{2}+a_{4} b_{4} c_{2}+a_{4} b_{4} d_{2}+a_{4} b_{4}+2 a_{4} c_{2} d_{2}+a_{4} c_{2} d_{4} \\
& +2 a_{4} c_{2}+a_{4} c_{4} d_{2}+a_{4} c_{4}+2 a_{4} d_{2}+a_{4} d_{4}+a_{6} b_{2} c_{2}+a_{6} b_{2} d_{2} \\
& +a_{6} c_{2} d_{2}+a_{6}+6 b_{2} c_{2} d_{2}+2 b_{2} c_{2} d_{4}+b_{2} c_{2} d_{6}+b_{2} c_{2}+2 b_{2} c_{4} d_{2} \\
& +b_{2} c_{4} d_{4}+2 b_{2} c_{4}+b_{2} c_{6} d_{2}+b_{2} d_{2}+2 b_{2} d_{4}+3 b_{2}+2 b_{4} c_{2} d_{2} \\
& +b_{4} c_{2} d_{4}+2 b_{4} c_{2}+b_{4} c_{4} d_{2}+b_{4} c_{4}+2 b_{4} d_{2}+b_{4} d_{4}+b_{6} c_{2} d_{2} \\
& +b_{6}+c_{2} d_{2}+2 c_{2} d_{4}+3 c_{2}+2 c_{4} d_{2}+c_{4} d_{4}+c_{6}+3 d_{2}+d_{6} \text {, }
\end{aligned}
$$




$$
\begin{aligned}
& \wedge^{7}\left(\rho_{U}\right)=\wedge^{9}\left(\rho_{U}\right)=7 a b c d+6 a b c d_{3}+3 a b c d_{5}+a b c d_{7}+6 a b c_{3} d+6 a b c_{3} d_{3} \\
& +2 a b c_{3} d_{5}+3 a b c_{5} d+2 a b c_{5} d_{3}+a b c_{7} d+6 a b_{3} c d+6 a b_{3} c d_{3} \\
& +2 a b_{3} c d_{5}+6 a b_{3} c_{3} d+4 a b_{3} c_{3} d_{3}+a b_{3} c_{3} d_{5}+2 a b_{3} c_{5} d \\
& +a b_{3} c_{5} d_{3}+3 a b_{5} c d+2 a b_{5} c d_{3}+2 a b_{5} c_{3} d+a b_{5} c_{3} d_{3}+a b_{7} c d \\
& +6 a_{3} b c d+6 a_{3} b c d_{3}+2 a_{3} b c d_{5}+6 a_{3} b c_{3} d+4 a_{3} b c_{3} d_{3} \\
& +a_{3} b c_{3} d_{5}+2 a_{3} b c_{5} d+a_{3} b c_{5} d_{3}+6 a_{3} b_{3} c d+4 a_{3} b_{3} c d_{3} \\
& +a_{3} b_{3} c d_{5}+4 a_{3} b_{3} c_{3} d+3 a_{3} b_{3} c_{3} d_{3}+a_{3} b_{3} c_{5} d+2 a_{3} b_{5} c d \\
& +a_{3} b_{5} c d_{3}+a_{3} b_{5} c_{3} d+3 a_{5} b c d+2 a_{5} b c d_{3}+2 a_{5} b c_{3} d \\
& +a_{5} b c_{3} d_{3}+2 a_{5} b_{3} c d+a_{5} b_{3} c d_{3}+a_{5} b_{3} c_{3} d+a_{7} b c d \text {, } \\
& \wedge^{8}\left(\rho_{U}\right)=10 a_{2} b_{2} c_{2} d_{2}+5 a_{2} b_{2} c_{2} d_{4}+a_{2} b_{2} c_{2} d_{6}+5 a_{2} b_{2} c_{2}+5 a_{2} b_{2} c_{4} d_{2} \\
& +2 a_{2} b_{2} c_{4} d_{4}+4 a_{2} b_{2} c_{4}+a_{2} b_{2} c_{6} d_{2}+a_{2} b_{2} c_{6}+5 a_{2} b_{2} d_{2}+4 a_{2} b_{2} d_{4} \\
& +a_{2} b_{2} d_{6}+4 a_{2} b_{2}+5 a_{2} b_{4} c_{2} d_{2}+2 a_{2} b_{4} c_{2} d_{4}+4 a_{2} b_{4} c_{2}+2 a_{2} b_{4} c_{4} d_{2} \\
& +a_{2} b_{4} c_{4}+4 a_{2} b_{4} d_{2}+a_{2} b_{4} d_{4}+a_{2} b_{4}+a_{2} b_{6} c_{2} d_{2}+a_{2} b_{6} c_{2} \\
& +a_{2} b_{6} d_{2}+a_{2} b_{6}+5 a_{2} c_{2} d_{2}+4 a_{2} c_{2} d_{4}+a_{2} c_{2} d_{6}+4 a_{2} c_{2}+4 a_{2} c_{4} d_{2} \\
& +a_{2} c_{4} d_{4}+a_{2} c_{4}+a_{2} c_{6} d_{2}+a_{2} c_{6}+4 a_{2} d_{2}+a_{2} d_{4}+a_{2} d_{6}+5 a_{4} b_{2} c_{2} d_{2} \\
& +2 a_{4} b_{2} c_{2} d_{4}+4 a_{4} b_{2} c_{2}+2 a_{4} b_{2} c_{4} d_{2}+a_{4} b_{2} c_{4}+4 a_{4} b_{2} d_{2}+a_{4} b_{2} d_{4} \\
& +a_{4} b_{2}+2 a_{4} b_{4} c_{2} d_{2}+a_{4} b_{4} c_{2}+a_{4} b_{4} c_{4}+a_{4} b_{4} d_{2}+a_{4} b_{4} d_{4}+2 a_{4} b_{4} \\
& +4 a_{4} c_{2} d_{2}+a_{4} c_{2} d_{4}+a_{4} c_{2}+a_{4} c_{4} d_{2}+a_{4} c_{4} d_{4}+2 a_{4} c_{4}+a_{4} d_{2} \\
& +2 a_{4} d_{4}+3 a_{4}+a_{6} b_{2} c_{2} d_{2}+a_{6} b_{2} c_{2}+a_{6} b_{2} d_{2}+a_{6} b_{2}+a_{6} c_{2} d_{2}+a_{6} c_{2} \\
& +a_{6} d_{2}+a_{8}+5 b_{2} c_{2} d_{2}+4 b_{2} c_{2} d_{4}+b_{2} c_{2} d_{6}+4 b_{2} c_{2}+4 b_{2} c_{4} d_{2} \\
& +b_{2} c_{4} d_{4}+b_{2} c_{4}+b_{2} c_{6} d_{2}+b_{2} c_{6}+4 b_{2} d_{2}+b_{2} d_{4}+b_{2} d_{6}+4 b_{4} c_{2} d_{2} \\
& +b_{4} c_{2} d_{4}+b_{4} c_{2}+b_{4} c_{4} d_{2}+b_{4} c_{4} d_{4}+2 b_{4} c_{4}+b_{4} d_{2}+2 b_{4} d_{4}+3 b_{4} \\
& +b_{6} c_{2} d_{2}+b_{6} c_{2}+b_{6} d_{2}+b_{8}+4 c_{2} d_{2}+c_{2} d_{4}+c_{2} d_{6}+c_{4} d_{2}+2 c_{4} d_{4} \\
& +3 c_{4}+c_{6} d_{2}+c_{8}+3 d_{4}+d_{8}+4 \text {, }
\end{aligned}
$$

where $a_{j}$ for instance denotes the jth symmetric power $S^{j}(a)=S^{j}\left(\rho_{a}\right)$ of $\rho_{a}$, the products denote tensor products, and sums denote the direct sums as before.

\section{Proof of Theorem 1}

In this section we give a proof of Theorem 1 stated in the previous section. To each representation $\mu$ of a complex semisimple Lie group $\mathscr{G}$ in $\mathbb{C}^{N}$ and an element $g \in \mathscr{G}$ we 
associate a polynomial $P_{\mu, g}(t)$ of degree $N$ in $t$ given by $P_{\mu, g}(t)=\operatorname{det}\left(1_{N}+\mu(g) t\right)$, where $1_{N}$ is the $N \times N$ identity matrix. Then we have

$$
P_{\mu, g}(t)=\sum_{k=1}^{N} \operatorname{tr}\left(\left(\wedge^{k} \mu\right) g\right) t^{k} .
$$

We also denote by

$$
P_{\mu}(t)=\operatorname{det}\left(1_{N}+\mu t\right)=\sum_{k=1}^{N} \operatorname{tr}\left(\wedge^{k} \mu\right) t^{k}
$$

the map that associates $P_{\mu, g}(t)$ to each $g \in \mathscr{G}$.

LEMMA 2. Let $\rho_{U}$ be the representation of $G_{U}=\left(S U_{2}\right)^{m}$ in $\mathbb{C}^{16}$ as described in Theorem 1. We fix an element $g=\left(g_{1}, \ldots, g_{m}\right)$ in $G_{U}=\left(S U_{2}\right)^{m}$ and assume that the eigenvalues of the $2 \times 2$ matrix $g_{d}$ are $\lambda$ and $\lambda^{-1}$. Then we have

$$
P_{\rho_{U}, g}(t)=P_{(a b c)_{U}, g}(\lambda t) P_{(a b c)_{U}, g}\left(\lambda^{-1} t\right),
$$

where $(a b c)_{U}$ is the representation of $G_{U}=\left(S U_{2}\right)^{m}$ induced by abc $=\rho_{a} \otimes \rho_{b} \otimes \rho_{c}$.

ProOF. Since $\lambda, \lambda^{-1}$ are the eigenvalues of $g_{d}$, we have

$$
g_{d}=v^{-1}\left(\begin{array}{cc}
\lambda & 0 \\
0 & \lambda^{-1}
\end{array}\right) v
$$

for some $v \in S U_{2}$ and $\lambda \in \mathbb{C}$. We have

$$
P_{\rho_{U}, g}(t)=\operatorname{det}\left(\left(\begin{array}{cc}
1_{N} & 0 \\
0 & 1_{N}
\end{array}\right)+v_{N}\left(\begin{array}{cc}
a b c(g) \lambda t & 0 \\
0 & a b c(g) \lambda^{-1} t
\end{array}\right) v_{N}^{-1}\right)
$$

where

$$
v_{N}=\left(\begin{array}{ll}
\alpha 1_{N} & \beta 1_{N} \\
\gamma 1_{N} & \delta 1_{N}
\end{array}\right) \quad \text { if } \quad v=\left(\begin{array}{ll}
\alpha & \beta \\
\gamma & \delta
\end{array}\right)
$$

Thus we have

$$
\begin{aligned}
P_{\rho_{i}, g}(t) & =\left(\begin{array}{cc}
1_{N}+a b c(g) \lambda t & 0 \\
0 & 1_{N}+a b c(g) \lambda^{-1} t
\end{array}\right) \\
& =\operatorname{det}\left(1_{N}+a b c(g) \lambda t\right) \operatorname{det}\left(1_{N}+a b c(g) \lambda^{-1} t\right) \\
& =P_{(a b c)_{U}, g}(\lambda t) P_{(a b c)_{U}, g}\left(\lambda^{-1} t\right) .
\end{aligned}
$$

Hence the lemma follows. 
From now on we shall denote the representation $\left(a_{j} b_{k} c_{l} d_{m}\right)_{U}$ of $G_{U}$ induced by the representation $a_{j} b_{k} c_{l} d_{m}$ of $G(\mathbb{C})$ simply by $a_{j} b_{k} c_{l} d_{m}$, where $j, k, l, m$ are nonnegative integers.

LEMMA 3. Using the notational convention in the previous paragraph, the exterior powers of the representations $a, a b$ and $a b c$ for $1 \leq a, b, c \leq m$ of $G_{U}=\left(S U_{2}\right)^{m}$ are as follows:

$$
\wedge^{0}(a)=\wedge^{2}(a)=1, \quad \wedge^{1}(a)=a .
$$

$$
\begin{aligned}
& \wedge^{0}(a b)=\wedge^{4}(a b)=1, \quad \wedge^{1}(a b)=\wedge^{3}(a b)=a b, \quad \wedge^{2}(a b)=a_{2}+b_{2} . \\
& \wedge^{0}(a b c)=\wedge^{8}(a b c)=1, \\
& \wedge^{1}(a b c)=\wedge^{7}(a b c)=a b c, \\
& \wedge^{2}(a b c)=\wedge^{6}(a b c)=a_{2} b_{2}+b_{2} c_{2}+c_{2} a_{2}+1, \\
& \wedge^{3}(a b c)=\wedge^{5}(a b c)=a_{3} b c+a b_{3} c+a b c_{3}+a b c, \\
& \wedge^{4}(a b c)=a_{4}+b_{4}+c_{4}+a_{2} b_{2} c_{2}+a_{2} b_{2}+b_{2} c_{2}+c_{2} a_{2}+1 .
\end{aligned}
$$

PROOF. See [6, Lemma 2.2.1].

Now we go back to the proof of Theorem 1. By Lemma 2, we have

$$
P_{\rho_{R} \cdot g}(t)=\sum_{k=0}^{16} \operatorname{tr}\left(\wedge^{k}\left(\rho_{R}\right)(g)\right) t^{k}=\left(\sum_{k=0}^{8} \operatorname{tr}\left(C_{k}(g)\right)(\lambda t)^{k}\right)\left(\sum_{k=0}^{8} \operatorname{tr}\left(C_{k}(g)\right)\left(\lambda^{-1} t\right)^{k}\right),
$$

where $C_{k}=\wedge^{k}(a b c)$ for each $k$ given by Lemma 3(iii). Since we are interested in the representations up to equivalence, from now on we shall identify representations with their traces. Thus we have

$$
\sum_{k=0}^{16} \wedge^{k}\left(\rho_{R}\right)(g) t^{k}=\left(\sum_{k=0}^{8} C_{k}(g)(\lambda t)^{k}\right)\left(\sum_{k=0}^{8} C_{k}(g)\left(\lambda^{-1} t\right)^{k}\right) .
$$

By comparing the coefficients of $t^{k}$ in the above relation, we obtain

$$
\begin{aligned}
\wedge^{0}\left(\rho_{U}\right)=\wedge^{16}\left(\rho_{U}\right)= & 1 \\
\wedge^{1}\left(\rho_{U}\right)=\wedge^{15}\left(\rho_{U}\right)= & a b c\left(\lambda+\lambda^{-1}\right) \\
\wedge^{2}\left(\rho_{U}\right)=\wedge^{14}\left(\rho_{U}\right)= & \left(a_{2} b_{2}+b_{2} c_{2}+c_{2} a_{2}+1\right)\left(\lambda^{2}+\lambda^{-2}\right)+a^{2} b^{2} c^{2} \\
\wedge^{3}\left(\rho_{U}\right)=\wedge^{13}\left(\rho_{U}\right)= & \left(a_{3} b c+a b_{3} c+a b c_{3}+a b c\right)\left(\lambda^{3}+\lambda^{-3}\right) \\
& +a b c\left(a_{2} b_{2}+b_{2} c_{2}+c_{2} a_{2}+1\right)\left(\lambda+\lambda^{-1}\right) \\
\wedge^{4}\left(\rho_{U}\right)=\wedge^{12}\left(\rho_{U}\right)= & \left(a_{4}+b_{4}+c_{4}+a_{2} b_{2} c_{2}+a_{2} b_{2}+b_{2} c_{2}+c_{2} a_{2}+1\right)\left(\lambda^{4}+\lambda^{-4}\right) \\
& +a b c\left(a_{3} b c+a b_{3} c+a b c_{3}+a b c\right)\left(\lambda^{2}+\lambda^{-2}\right) \\
& +\left(a_{2} b_{2}+b_{2} c_{2}+c_{2} a_{2}+1\right)^{2}
\end{aligned}
$$




$$
\begin{aligned}
& \wedge^{5}\left(\rho_{U}\right)=\wedge^{11}\left(\rho_{U}\right)=\left(a_{3} b c+a b_{3} c+a b c_{3}+a b c\right)\left(\lambda^{5}+\lambda^{-5}\right) \\
& +a b c\left(a_{4}+b_{4}+c_{4}+a_{2} b_{2} c_{2}+a_{2} b_{2}+b_{2} c_{2}+c_{2} a_{2}+1\right) \\
& \times\left(\lambda^{3}+\lambda^{-3}\right) \\
& +\left(a_{2} b_{2}+b_{2} c_{2}+c_{2} a_{2}+1\right)\left(a_{3} b c+a b_{3} c+a b c_{3}+a b c\right) \\
& \times\left(\lambda+\lambda^{-1}\right) \\
& \wedge^{6}\left(\rho_{U}\right)=\wedge^{10}\left(\rho_{U}\right)=\left(a_{2} b_{2}+b_{2} c_{2}+c_{2} a_{2}+1\right)\left(\lambda^{6}+\lambda^{-6}\right) \\
& +a b c\left(a_{3} b c+a b_{3} c+a b c_{3}+a b c\right)\left(\lambda^{4}+\lambda^{-4}\right) \\
& +\left(a_{2} b_{2}+b_{2} c_{2}+c_{2} a_{2}+1\right) \\
& \times\left(a_{4}+b_{4}+c_{4}+a_{2} b_{2} c_{2}+a_{2} b_{2}+b_{2} c_{2}+c_{2} a_{2}+1\right) \\
& \times\left(\lambda^{2}+\lambda^{-2}\right) \\
& +\left(a_{3} b c+a b_{3} c+a b c_{3}+a b c\right)^{2} \\
& \wedge^{7}\left(\rho_{U}\right)=\wedge^{9}\left(\rho_{U}\right)=a b c\left(\lambda^{7}+\lambda^{-7}\right)+a b c\left(a_{2} b_{2}+b_{2} c_{2}+c_{2} a_{2}+1\right)\left(\lambda^{5}+\lambda^{-5}\right) \\
& +\left(a_{2} b_{2}+b_{2} c_{2}+c_{2} a_{2}+1\right)\left(a_{3} b c+a b_{3} c+a b c_{3}+a b c\right) \\
& \times\left(\lambda^{3}+\lambda^{-3}\right) \\
& +\left(a_{3} b c+a b_{3} c+a b c_{3}+a b c\right) \\
& \times\left(a_{4}+b_{4}+c_{4}+a_{2} b_{2} c_{2}+a_{2} b_{2}+b_{2} c_{2}+c_{2} a_{2}+1\right) \\
& \times\left(\lambda+\lambda^{-1}\right) \\
& \wedge^{8}\left(\rho_{U}\right)=\left(\lambda^{8}+\lambda^{-8}\right)+(a b c)^{2}\left(\lambda^{6}+\lambda^{-6}\right) \\
& +\left(a_{2} b_{2}+b_{2} c_{2}+c_{2} a_{2}+1\right)^{2}\left(\lambda^{4}+\lambda^{-4}\right) \\
& +\left(a_{3} b c+a b_{3} c+a b c_{3}+a b c\right)^{2}\left(\lambda^{2}+\lambda^{-2}\right) \\
& +\left(a_{4}+b_{4}+c_{4}+a_{2} b_{2} c_{2}+a_{2} b_{2}+b_{2} c_{2}+c_{2} a_{2}+1\right)^{2} \text {. }
\end{aligned}
$$

By the Clebsch-Gordon formula we have

$$
\begin{aligned}
a_{k} \otimes a_{l} & =S^{k}\left(\rho_{a}\right) \otimes S^{l}\left(\rho_{a}\right) \\
& =S^{k+l}\left(\rho_{a}\right) \oplus S^{k+l-2} \oplus \cdots \oplus S^{|k-l|}\left(\rho_{a}\right) \\
& =a_{k+l} \oplus a_{k+l-2} \oplus \cdots \oplus a_{|k-l|},
\end{aligned}
$$

where $K, l$ are non-negative integers. Thus, using our notational convention, we have

$$
a_{k} a_{l}=a_{k+l}+a_{k+l-2}+\cdots+a_{|k-l|}
$$

Similar formulas are obtained for $b, c$ and $d$. 
LEMMA 4. If $\lambda$ and $\lambda^{-1}$ are the eigenvalues of $g_{d}$ as before, then we have

$$
\lambda^{n}+\lambda^{-n}=d_{n}-d_{n-2}
$$

for all $n \geq 2$.

PROOF. We use induction on $n$. Since $d^{2}=d_{2}+1$ by the Clebsch-Gordon formula, it follows that

$$
\lambda^{2}+\lambda^{-2}=\left(\lambda+\lambda^{-1}\right)^{2}-2=d^{2}-2=d_{2}-1
$$

hence the statement is true for $n=2$. Assuming that it is true for all $k \leq n$, we have

$$
\begin{aligned}
\lambda^{n+1}+\lambda^{-(n+1)} & =\left(\lambda^{n}+\lambda^{-n}\right)\left(\lambda+\lambda^{-1}\right)-\left(\lambda^{n-1}+\lambda^{-(n-1)}\right) \\
& =\left(d_{n}-d_{n-2}\right) d-\left(d_{n-1}-d_{n-3}\right) \\
& =d_{n} d-d_{n-2} d-d_{n-1}+d_{n-3} \\
& =d_{n+1}+d_{n-1}-\left(d_{n-1}+d_{n-3}\right)-d_{n-1}+d_{n-3} \\
& =d_{n+1}-d_{n-1}
\end{aligned}
$$

So the statement is true for $n+1$ and the lemma follows.

To complete the proof of Theorem 1 we first use Lemma 4 to replace the expressions of the form $\left(\lambda^{k}+\lambda^{-k}\right)$ in the relations for $\wedge^{0}(R), \ldots, \wedge^{16}(R)$ above by $d_{k}-d_{k-2}$, and then use the Clebsch-Gordon formula with the aid of a computer to obtain the formulas given in Theorem 1.

\section{Kuga fiber varieties}

In this section, we review the construction of Kuga fiber varieties over arithmetic varieties. Let $V$ be a vector space of dimension $2 n$ over $\mathbb{Q}$, and let $L$ be a lattice in $V$. Let $\beta$ be a nondegenerate alternating bilinear form on $V$ such that $\beta(L, L) \subset \mathbb{Z}$. Let

$$
S p(V, \beta)=\{g \in G L(V) \mid \beta(g x, g y)=\beta(x, y) \text { for all } x, y \in V\}
$$

be the symplectic group of the pair $(V, \beta)$, and let $\mathscr{H}$ denote the Siegel half space

$$
\begin{array}{r}
\mathscr{H}=\left\{J \in G L(V(\mathbb{R})) \mid J^{2}=-1, \quad \beta(x, J y)\right. \text { is a positive definite } \\
\text { symmetric bilinear form in } x, y \in V(\mathbb{R})\} .
\end{array}
$$

Then each element $J \in \mathscr{H}$ defines a complex structure on $V(\mathbb{R})$ and there is a unique complex analytic structure on $\mathscr{H} \times V(\mathbb{R})$ such that the projection $P: \mathscr{H} \times V(\mathbb{R}) \rightarrow$ 
$\mathscr{H}$ is a complex vector bundle over $\mathscr{H}$. For each $J$ if we denote the complex vector space $(V(\mathbb{R}), J)$ by $V_{J}$, then the complex torus $A_{J}=V_{J} / L$ is an abelian variety with the polarization $\beta$. We set

$$
A_{\mathscr{H}}=L \backslash \mathscr{H} \times V(\mathbb{R}),
$$

where the action of $L$ on $\mathscr{H} \times V(\mathbb{R})$ is given by

$$
l \cdot(J, v)=(J, v+l) \quad \text { for } \quad J \in \mathscr{H}, v \in V(\mathbb{R}) \text { and } l \in L .
$$

Then the vector bundle $P: \mathscr{H} \times V(\mathbb{R}) \rightarrow \mathscr{H}$ induces the fiber bundle $\pi_{\mathscr{H}}:$ $A_{\mathscr{H}} \rightarrow \mathscr{H}$ whose fibers are abelian varieties polarized by $\beta$. We set

$$
S p(L, \beta)=\{g \in \operatorname{Sp}(V, \beta) \mid g L=L\},
$$

and take a subgroup $\Gamma_{0}$ of $S p(L, \beta)$ of finite index that contains no elements of finite order. Then the quotient $X_{0}=\Gamma_{0} \backslash \mathscr{H}$ is an arithmetic variety that can be considered as a Zariski open subset of a complex projective variety. Now the fiber bundle $\pi_{\mathscr{H}}: A_{\mathscr{H}} \rightarrow \mathscr{H}$ induces the standard family of abelian varieties $\pi_{0}: Y_{0} \rightarrow X_{0}$ over $X_{0}$.

Let $\tilde{G}$ be a semisimple algebraic group defined over $\mathbb{Q}$, and let $\tilde{K}$ be a maximal compact subgroup of the semisimple Lie group $\tilde{G}(\mathbb{R})$. We assume that the symmetric space $\tilde{D}=\tilde{G}(\mathbb{R}) / \tilde{K}$ has a $\tilde{G}(\mathbb{R})$-invariant complex structure. Let $\tilde{\Gamma} \subset \tilde{G}(\mathbb{Q})$ be a torsion-free cocompact arithmetic subgroup $\tilde{G}$, and let $\tilde{X}=\tilde{\Gamma} \backslash \tilde{D}$ be the corresponding arithmetic variety. Let $\tilde{\rho}: \tilde{G} \rightarrow S p(V, \beta)$ be a symplectic representation and $\tilde{\tau}: \tilde{D} \rightarrow \mathscr{H}$ a holomorphic map such that $\tilde{\rho}(\tilde{\Gamma}) \subset \Gamma_{0}$ and

$$
\tilde{\tau}(g y)=\tilde{\rho}(g) \tilde{\tau}(y) \text { for all } g \in \tilde{G}(\mathbb{R}) \text { and } y \in \tilde{D} .
$$

Then the pair $(\tilde{\rho}, \tilde{\tau})$ determines a fiber variety $\tilde{\pi}: \tilde{Y} \rightarrow \tilde{X}$ over the arithmetic variety $\tilde{X}$ whose fibers are abelian varieties called a Kuga fiber variety. It is constructed as follows. The semidirect product $\tilde{\Gamma} \times_{\tilde{\rho}} L$ with respect to the representation $\tilde{\rho}: \tilde{\Gamma} \rightarrow$ $\operatorname{Aut}(L)$ operates on the product manifold $\tilde{D} \times V(\mathbb{R})$ properly discontinuously by

$$
(\gamma, l) \cdot(y, v)=(\gamma y, \gamma v+l)
$$

for $(\gamma, l) \in \tilde{\Gamma} \ltimes_{\rho} L$ and $(y, v) \in \tilde{D} \times V(\mathbb{R})$. We set $\tilde{Y}=\tilde{\Gamma} \ltimes_{\tilde{\rho}} L \backslash \tilde{D} \times V(\mathbb{R})$, and denote by $\tilde{\pi}$ the natural projection of $\tilde{Y}$ onto $\tilde{X}=\tilde{\Gamma} \backslash \tilde{D}$. Then $\tilde{\pi}: \tilde{Y} \rightarrow \tilde{X}$ is a fiber bundle over $\tilde{X}$, which is in fact the pullback of the standard fiber bundle $\pi_{0}: Y_{0} \rightarrow X_{0}$ via the map $\tilde{X} \rightarrow X_{0}$ induced by $\tilde{\tau}: \tilde{D} \rightarrow \mathscr{H}$. It is known that $\tilde{Y}$ has a structure of a complex projective variety and that the fiber $\tilde{Y}_{x}$ over each $x \in \tilde{X}$ is an abelian variety polarized by $\beta$. Such a fiber variety $\tilde{\pi}: \tilde{Y} \rightarrow \tilde{X}$ is called a Kuga fiber variety (see $[4$, 8], [9, Chapter 4]). 


\section{Hodge cycles}

In this section we consider Hodge cycles on generic fibers of Kuga fiber varieties associated to quaternion algebras and prove the main theorem of the paper. Let $G$ be the algebraic group defined over $\mathbb{Q}$ considered in Section 1 . Thus $G$ is the algebraic group $\operatorname{Res}_{K / Q}\left(S L_{1}(B)\right)$ where $B$ is a quaternion algebra over a totally real number field with $[k: \mathbb{Q}]=m$. Let $\rho: G \rightarrow S p(V, \beta)$ with $V=\mathbb{C}^{8}$ be a symplectic representation of $G$ associated to a subset $R=\left\{\varphi_{a}, \varphi_{b}, \varphi_{c}, \varphi_{d}\right\}$ of $S$ as in Section 1, and let $\Gamma$ be a torsion free arithmetic subgroup of $G$ with $\rho(\Gamma) \subset \Gamma_{S}$. Let $\tau: D \rightarrow \mathscr{H}$ be a holomorphic map such that $\rho$ and $\tau$ are equivariant, and let $\phi: X \rightarrow X_{0}$ be the morphism of varieties induced by $\tau$. By pulling back the fiber bundle $\pi_{0}: Y_{0} \rightarrow X_{0}$ via the morphism $\phi: X \rightarrow X_{0}$, we obtain the Kuga fiber variety $\pi: Y \rightarrow X$ over the arithmetic variety $X$.

We fix a generic point $x$ in $X$, and identify $\Gamma$ with the fundamental group $\pi_{1}(X, x)$ of $X$ at $x$. We also identify the fiber $Y_{x}$ of $Y$ over $x \in X$ with $V / L$, which induces the following further identifications:

$$
H_{1}\left(Y_{x}, \mathbb{Q}\right)=L \otimes \mathbb{Q}=V, \quad H_{k}\left(Y_{x}, \mathbb{Q}\right)=\wedge^{k}(V), \quad H^{k}(Y, \mathbb{Q})=\wedge^{k}(V)^{*} ;
$$

here $*$ denotes the dual of the vector space. The action of $\pi_{1}(X, x)$ on $H^{k}\left(Y_{x}, \mathbb{Q}\right)$ corresponds to the action $\wedge^{k}\left(\rho^{*}\right)$ of $\Gamma$ on $\wedge^{k}(V)^{*}$; hence we have

$$
H^{k}\left(Y_{x}, \mathbb{Q}\right)^{\pi_{1}(X, x)}=\left(\wedge^{k}(V)^{*}\right)^{\Gamma}
$$

DEFINITION. Let $\pi: Y \rightarrow X$ be a Kuga fiber variety associated to $\rho: G \rightarrow$ $S p(V, \beta)$ and $\tau: D \rightarrow \mathscr{H}$, and let $g(\mathbb{R})$ be the Lie algebra of $G(\mathbb{R})$. The Kuga fiber variety $(Y, \pi)$ is of inner type if there is a map $r: D \rightarrow \mathfrak{g}(\mathbb{R})$ such that

$$
\cos (\pi t / 2) I+\sin (\pi t / 2) \tau(x) I=\rho(\exp (r(x) t))
$$

for $x \in D$ and $t \in \mathbb{R}$, where $I$ is the identity map on $V$.

REMARK 5.1. A Kuga fiber variety that does not allow deformations is said to be rigid. Any rigid Kuga fiber variety is of inner type. For example, if $R=$ $\left\{\varphi_{a}, \varphi_{b}, \varphi_{c}, \varphi_{d}\right\} \subset S$ is $\operatorname{Gal}(K / \mathbb{Q})$-invariant and if $\left|R \cap S_{0}\right|=1$, then the Kuga fiber variety associated to $R$ is rigid and therefore of inner type (see [5]).

We shall denote by $H H^{2 k}\left(Y_{x}, \mathbb{Q}\right)$ the space of Hodge cycles of codimension $k$ in $Y_{x}$, that is,

$$
H H^{2 k}\left(Y_{x}, \mathbb{Q}\right)=H^{(k, k)}\left(Y_{x}\right) \cap H^{2 k}\left(Y_{x}, \mathbb{Q}\right) .
$$

The Hodge conjecture states that the space $H H^{2 k}\left(Y_{x}, \mathbb{Q}\right)$ coincides with the space of algebraic cycles of codimension $k$ for $0 \leq k \leq \operatorname{dim}_{\mathbb{C}} Y_{x}$. 
PROPOSITION 5.1. Let $Y_{x}$ be a generic fiber over $x \in X$ of a Kuga fiber variety $\pi: Y \rightarrow X$ of inner type. Then

$$
H H^{2 k}\left(Y_{x}, \mathbb{Q}\right)=H^{2 k}\left(Y_{x}\right)^{\pi_{1}(X, x)}
$$

for all even integers $k$ with $0 \leq k \leq \operatorname{dim}_{\mathbb{C}} Y_{x}$.

PROOF. See [10].

Now we state the main theorem of the paper about the Hodge cycles on Kuga fiber varieties associated to quaternion algebras.

THEOREM 5.2. Let $Y_{x}$ be a generic fiber over $x \in X$ of a Kuga fiber variety $\pi: Y \rightarrow X$ of inner type associated to the quaternion algebra $B$ in Section 2 and the pair $(\rho, \tau)$. Then we have

$$
\begin{aligned}
& \operatorname{dim} H H^{0}\left(Y_{x}, \mathbb{Q}\right)=\operatorname{dim} H H^{16}\left(Y_{x}, \mathbb{Q}\right)=1, \\
& \operatorname{dim} H H^{2}\left(Y_{x}, \mathbb{Q}\right)=\operatorname{dim} H H^{14}\left(Y_{x}, \mathbb{Q}\right)=0, \\
& \operatorname{dim} H H^{4}\left(Y_{x}, \mathbb{Q}\right)=\operatorname{dim} H H^{12}\left(Y_{x}, \mathbb{Q}\right)=3, \\
& \operatorname{dim} H H^{6}\left(Y_{x}, \mathbb{Q}\right)=\operatorname{dim} H H^{10}\left(Y_{x}, \mathbb{Q}\right)=0, \\
& \operatorname{dim} H H^{8}\left(Y_{x}, \mathbb{Q}\right)=4 .
\end{aligned}
$$

Proof. Since $\Gamma$ is Zariski-dense in $G$, the action $\wedge^{k}\left(\rho^{*}\right)$ of $\Gamma$ in $\wedge^{k}(V)^{*}$ can be extended to the action $\wedge^{k}\left(\rho^{*}\right)$ of $G(\mathbb{C})$ on $\wedge^{k}(V(\mathbb{C}))^{*}$. Thus we have

$$
\left(\wedge^{k}(V(\mathbb{C}))^{*}\right)^{\Gamma}=\left(\wedge^{k}(V(\mathbb{C}))^{*}\right)^{G(\mathbb{C})}
$$

On the other hand, by the unitary trick, we have

$$
\left(\wedge^{k}(V(\mathbb{C}))^{*}\right)^{G(\mathbb{C})}=\left(\wedge^{k}(V(\mathbb{C}))^{*}\right)^{G_{U}}
$$

where $G_{U}=\left(S U_{2}\right)^{m}$ is the compact real form of $G(\mathbb{C})=S L_{2}(\mathbb{C})^{m}$ as in Theorem 1 . Hence it follows that

$$
\operatorname{dim} H H^{2 k}\left(Y_{x}, \mathbb{Q}\right)=\operatorname{dim}_{\mathbb{C}}\left(H^{2 k}\left(Y_{x}\right)^{\pi_{1}(X, x)}\right)=\operatorname{dim}_{\mathbb{C}}\left(\wedge^{k}(V(\mathbb{C}))^{*}\right)^{G_{U}} .
$$

Since the symplectic representation $\rho$ is equivalent to its dual $\rho^{*}$, we have

$$
\operatorname{dim}_{\mathbb{C}}\left(\wedge^{k}(V(\mathbb{C}))^{*}\right)^{G_{U}}=\int_{G_{U}} \operatorname{tr}\left(\wedge^{2 k}\left(\rho_{U}\right)\right)(g) d g
$$


where $d g$ is the Haar measure of $G_{U}$ normalized by $\int_{G_{U}} d g=1$. On the other hand, the integral

$$
\int_{G_{U}} \operatorname{tr}\left(\wedge^{2 k}\left(\rho_{U}\right)\right)(g) d g
$$

is equal to the multiplicity $M_{2 k}=\left(\wedge^{2 k}\left(\rho_{U}\right): 1\right)$ of the trivial representation 1 in the representation $\wedge^{2 k}\left(\rho_{U}\right)$. By Theorem 1 we have

$$
M_{0}=M_{16}=1, \quad M_{2}=M_{14}=0, \quad M_{4}=M_{12}=3, \quad M_{6}=M_{10}=0, \quad M_{8}=4 ;
$$

hence the theorem follows.

\section{References}

[1] S. Abdulali, 'Field of definition for some Hodge cycles', Math. Ann. 285 (1989), 289-295.

[2] S. Addington, 'Equivariant holomorphic maps of symmetric domains', Duke Math. J. 55 (1987), 65-88.

[3] B. Gordon, 'Topological and algebraic cycles in Kuga-Shimura varieties', Math. Ann. 279 (1988), 395-402.

[4] M. Kuga, Fiber varieties over a symmetric space whose fibers are abelian varieties I, II, Lecture Notes (Univ. of Chicago Press, Chicago, 1963/64).

[5] —-, 'Algebraic cycles in gtabv', J. Fac. Sci. Univ. of Tokyo Sect. IA Math. 29 (1982), 13-29.

[6] - 'Chemistry and GTABVs', Progr. Math. 46 (1984), 269-281.

[7] —_, 'Invariants and Hodge cycles', Adv. Stud. Pure Math. 15 (1989), 373-413.

[8] M. H. Lee, 'Conjugates of equivariant holomorphic maps of symmetric domains', Pacific J. Math. 149 (1991), 127-144.

[9] I. Satake, Algebraic structures of symmetric domains (Princeton Univ. Press, Princeton, 1980).

[10] M. C. Tjiok, Algebraic cycles in a certain fiber variety ( $\mathrm{PhD}$. Thesis, SUNY at Stony Brook, 1980).

Department of Mathematics

University of Northern Iowa

Cedar Falls, Iowa 50614

USA

e-mail address: lee@math.uni.edu 